\title{
Regulación de la producción forestal con pronósticos de modelación Clutter y redes neuronales artificiales en plantaciones de eucalipto del Estado de Minas Gerais - Brasil
}

\section{Regulation of forest production with modeling forecasts Clutter and artificial neural networks in eucalyptus plantations in the State of Minas Gerais - Brazil}

\author{
*Gianmarco Goycochea Casas ${ }^{1}$, Juan Rodrigo Baselly Villanueva ${ }^{2}$, Helio Garcia Leite ${ }^{3}$, Ana Carolina de \\ Albuquerque Santos ${ }^{4}$, Carlos Alberto Araujo Júnior ${ }^{5}$, Aline Edwiges Mazon de Alcántara ${ }^{6}$
}

\begin{abstract}
RESUMEN
El presente estudio fue realizado con los objetivos de regular la producción forestal con pronósticos de modelación Clutter y Redes Neuronales Artificiales (RNA) en plantaciones de eucalipto (híbridos de Eucalyptus urophylla x Eucalyptus grandis), localizado en la región Centro Oeste del Estado de Minas Gerais, Brasil. De las tablas de producción se generaron procesos de regulación forestal en formato de programación lineal utilizando el software RPF 2.0®, con el fin de maximizar el ingreso líquido futuro para 22 años, 2016-2038. Se generaron dos planos de manejo, obteniendo variables de prescripción, edad, rotación, volumen, costos, demanda. Para la aplicación del modelo de programación lineal se consideró un horizonte de planeamiento de 22 periodos, tasa de interés de $11,5 \%$ y una edad regulatoria de 7 años con $0 \%$ de variación permitida, considerando una sola rotación y una variación de producción entre $100000 \mathrm{~m}^{3}$ y $60000 \mathrm{~m}^{3}$. Se concluye que los dos planos de proyección tienen influencia en la regulación forestal, destacando la técnica de RNA como una alternativa más real y confiable para regular una plantación.
\end{abstract}

Palabras clave: Redes neuronales artificiales (RNA), eucalipto, regulación forestal.

\begin{abstract}
The present study was carried out with the objectives of regulating forest production with forecasts of Clutter modeling and Artificial Neural Networks (RNA) in eucalyptus plantations (Eucalyptus urophylla $\mathrm{x}$ Eucalyptus grandis hybrids), located in the Midwest region of the State of Minas Gerais, Brazil. From the production tables, forest regulation processes were generated in a linear programming format using RPF $2.0 \AA$ software, in order to maximize the future net income for 22 years, 2016-2038. Two plans were generated by projection of management, obtaining variables of prescription, age, turnover, volume, costs, demand. For the application of the linear programming model, a planning horizon of 22 periods, an interest rate of $11.5 \%$ and a regulatory age of 7 years with $0 \%$ of allowable variation was considered, and a single rotation and a production variation between 100000 $\mathrm{m}^{3}$ and $60000 \mathrm{~m}^{3}$. It is concluded that the two projection planes have influence in the forest regulation, emphasizing the RNA technique as a more real and reliable alternative to regulate a plantation.
\end{abstract}

Keywords: ANN, artificial neural networks, eucalyptus, system of equations.

\footnotetext{
${ }^{1 *}$ Egresado Ingeniería Forestal. Universidad Nacional de Cajamarca, Sede Jaén. Cajamarca, Perú. Correo electrónico: ggoycocheac50@gmail.com

${ }^{2}$ Egresado de Ingeniería Forestal. Universidad Nacional de Cajamarca, Sede Central. Cajamarca, Perú.

${ }^{3}$ Profesor del Departamento de Ingeniería Forestal. Universidade Federal de Viçosa. Viçosa MG, Brasil.

${ }^{4 y 5}$ Estudiante de doctorado del Departamento de Ingeniería Forestal. Universidade Federal de Viçosa. Viçosa MG, Brasil.

${ }^{6}$ CENIBRA - Celulose Nipo-Brasileira S.A.
} 


\section{INTRODUCCIÓN}

Las Redes Neuronales Artificiales (RNA) son redes de neuronas artificiales distribuido en paralelo (en capas) y conectadas entre sí de una manera específica para ejecutar una determinada tarea (Bullinaria, 2014).

Según Clutter et al. (1983), el real papel del manejador forestal es la gerencia, de modo inteligente. Para desempeñar las funciones administrativas, los gestores forestales recurren a otras áreas científicas como economía, matemática y estadística; tales disciplinas direccionan la toma de decisiones a largo plazo (Magalhães, 2014); las RNA pueden ser consideradas como una de las promotoras para dicha finalidad.

Una plantación regulada es aquella en que son observadas áreas proporcionales en todas las clases de edad, creciendo constantemente, siendo capaces de proporcionar materia prima en cantidad y calidad suficientes para atender al mercado de forma continua (Davis y Johnson, 1987).

Los dos principales modelos utilizados para la regulación de la producción forestal son conocidos como el modelo I y modelo II de Johnson y Scheurman (1977).

El desarrollo de modelos de regulación de la producción es realizado utilizando métodos numéricos como programación lineal, programación multiobjetivo, programación entera, programación entera mixta, programación dinámica, meta heurística y simulación (Binoti, 2010).

En empresas de base forestal, la demanda periódica de madera es la principal restricción en los problemas de programación matemática, es por ello, el modelamiento busca, predecir volúmenes para garantizar el abastecimiento regular a las industrias (Dykstra, 1984; Leuschner, 1984; Buongiorno y Gilless, 2003).

Se han realizado muchos trabajos de modelamiento orientados a la regulación de la producción forestal, empleando el tradicional modelo de Clutter y recientemente se está introduciendo las RNA (Rodrigues, 2001; Castro, 2007; Binoti, 2010; Cavalcante, 2011; Monte, 2012, Da Silva, 2014; Rode, 2015, De Carvalho, 2015).

El objetivo del presente estudio fue regular la producción forestal con pronósticos de modelación Clutter y RNA en plantaciones de eucalipto en el Estado de Minas Gerias, Brasil.

\section{MATERIAL Y MÉTODOS}

Los datos de inventarios fueron cedidos por la empresa Celulose Nipo-Brasileira S.A, localizado en la región Centro Oeste del Estado de Minas Gerais, Brasil, donde las variables son: catastro actual 2016, financiero (costos de silvicultura, acumulado, transporte, colecta y demanda) y productividad (Clutter y RNA) en poblaciones de híbridos de Eucalyptus urophylla $\mathrm{x}$ Eucalyptus grandis.

Se generó y procesó al modelo de regulación forestal en formato de programación lineal utilizando el software RPF 2.0® (Binoti, 2012) con el fin de maximizar el ingreso líquido futuro. Para ello, se aplicó la programación matemática en modelos de regulación forestal que son atribuidas por Johnson y Sheurman:

$$
\begin{gathered}
\text { Max.Z }=\sum_{i=1}^{M} \sum_{j=1}^{N} C i j X i j \\
\mathrm{Cij}=\frac{\sum_{K=0}^{H-1} P i j k-C P(1+\mathrm{i})^{I i j k}}{(1+\mathrm{i})^{k}} \\
+\frac{D_{i j H} V_{i j H}-C P(1+\mathrm{i})^{I i j H}}{(1+\mathrm{r})^{H}}
\end{gathered}
$$

Dónde: Max. $Z$ = maximización del valor presente liquido global de la plantación forestal; $\mathrm{Cij}=$ valor presente liquido de cada hectárea de clase de edades $i$, manejado sobre la alternativa de manejo $\mathrm{j}$; Xij = fracción de área por hectárea de clases de edades $\mathrm{i}$ asimilada a la prescripción de manejo j; $\mathrm{M}=$ número total de clases de edades; $\mathrm{N}=$ número total de alternativas de manejo de la i-ésima clase de edad; Pijk = precio por unidad de volumen de madera comercial; Vijk = volumen comercial, en $\mathrm{m}^{3} /$ ha; $\mathrm{DijH}$ $=$ volumen no comercial, en $\mathrm{m}^{3} / \mathrm{ha}$; IijH $=$ edad de árboles, en años, al final del horizonte de planeamiento; Iijk = edad de árboles, en años, de las clases edades $\mathrm{i}$ en el inicio del periodo $\mathrm{k} ; \mathrm{CP}=$ costo de la plantación; $i=$ tasa de interés anual.

Para el control sobre el flujo de la producción forestal durante el horizonte de planeamiento será impuesto usando las siguientes expresiones:

$$
\begin{aligned}
& V_{\mathrm{H}(\mathrm{k}+1)} \geq(1-\underline{\alpha}) \mathrm{V}_{\mathrm{HK}} \\
& \mathrm{V}_{\mathrm{H}(\mathrm{k}+1)} \leq(1+\beta) \mathrm{V}_{\mathrm{HK}}
\end{aligned}
$$

Dónde: Vijk = volumen, en $\mathrm{m}^{3} / \mathrm{ha}$, producido por la iésima clase de edad sujeta a la j-ésima alternativa de manejo en el inicio del periodo $\mathrm{k}$; Vhk = volumen total removido en el periodo $\mathrm{k}$. 
Para la regulación al final de horizonte de planeamiento consiste en la inclusión de las restricciones que impongan una distribución adecuada de clases de edades para el inventario final, así es posible tener una plantación regulada al final de un horizonte de planeamiento finito, usando la siguiente expresión:

$$
\sum_{i=1}^{M} \sum_{j=1}^{N} X i j k=\frac{S}{N C}
$$

Dónde: Xijk = área de la i-ésima clase de edad manejada sobre la j-ésima alternativa de manejo, cuyas áreas tendrán $\mathrm{k}$ periodos (años) de edad. $\mathrm{S}=$ área total de la plantación, en ha; $\mathrm{NC}=$ número de clases de edad para la plantación regulada.

\section{RESULTADOS}

La formulación del modelo de programación lineal y las tablas de producción obtenidas por la técnica de modelaje de Clutter y RNA, generaron dos planos de manejo, obteniéndose variables de prescripción, edad, rotación, volumen, costos, demanda; que fueron interactuadas en el software RPF $2.0 \AA$. En el Software se colocaron los datos cedidos por la empresa forestal, para la aplicación del modelo de programación lineal se consideró un horizonte de planeamiento de 22 periodos, tasa de interés de $11,5 \%$ y una edad regulatoria de 7 años con $0 \%$ de variación permitida, considerando una sola rotación y una variación de producción entre $100000 \mathrm{~m}^{3}$ y 60 $000 \mathrm{~m}^{3}$. El programa permitió visualizar las alternativas de manejo como sus cálculos elaborados por el sistema y las tablas de producción generadas a partir de las diferentes técnicas de pronóstico de la producción (Clutter - RNA) interfiriendo no solo en el valor de rendimiento acumulado, sino también en la producción total anual de la floresta regulada.

En las Figuras 1, 2, 3 y 4, se pueden apreciar la planificación de la regulación forestal generada por la producción del modelo de Clutter y RNA por 22 años (2016 - 2038), especificando la producción, área de cosecha, edad media de corte e ingreso acumulado prescrita por los modelos de regulación.

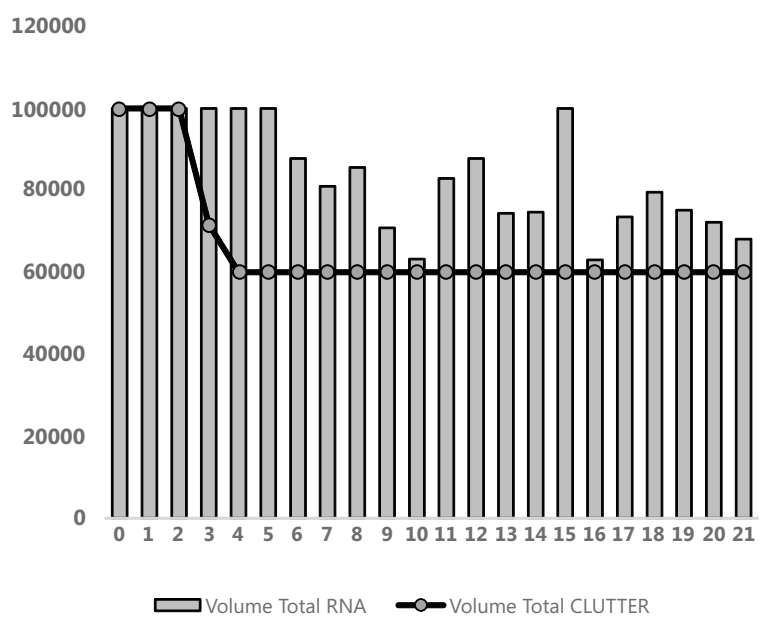

Figura 1. Producción total prescrita por los modelos de regulación generados con los resultados de dos metodologías para proyección de producción, considerándose como la función objetivo en maximizar el ingreso líquido

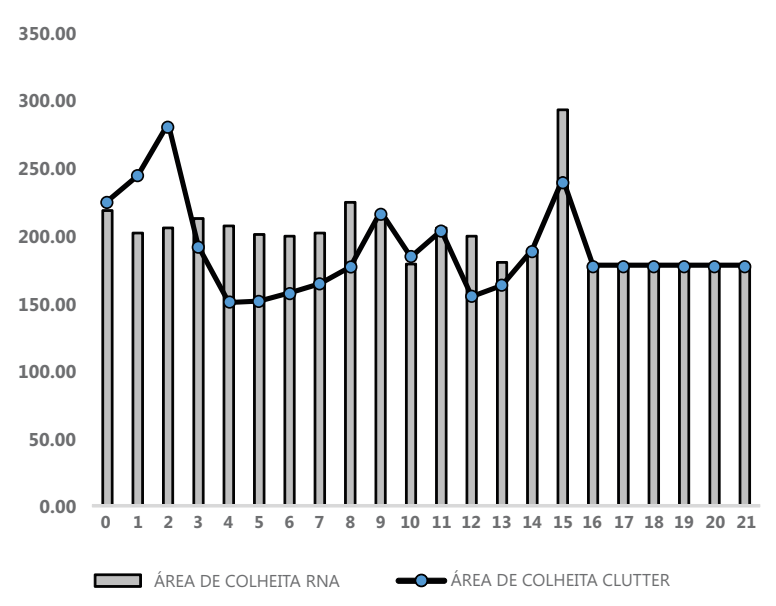

Figura 2. Área de cosecha prescrita por los modelos de regulación generados con los resultados de dos metodologías para proyección de producción, considerándose como la función objetivo en maximizar el ingreso líquido 


\section{Regulación de la producción forestal}

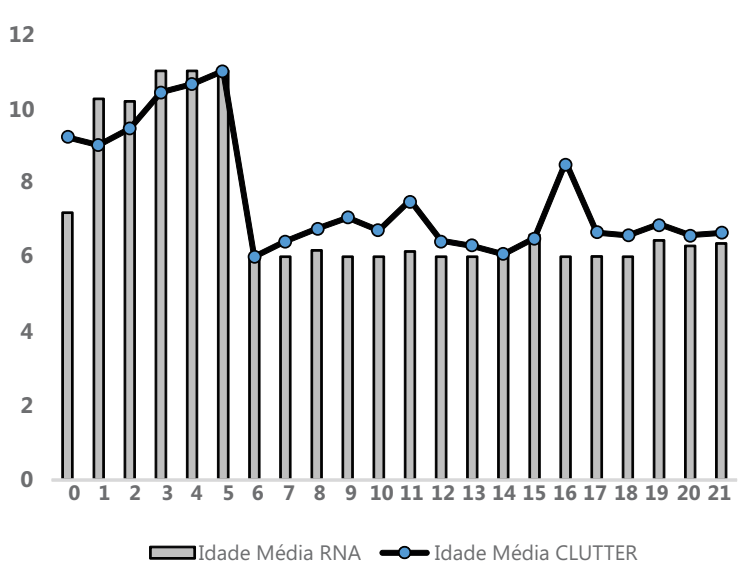

Figura 3. Edad media prescrita por los modelos de regulación generados con los resultados de dos metodologías para proyección de producción, considerándose como la función objetivo en maximizar el ingreso líquido

\section{DISCUSIÓN}

El volumen proyectado por el modelo de Clutter es más uniforme, pero tiene menor precisión en las proyecciones que las RNA; Da silva (2005) indica que los usos de las técnicas de proyección de volumen llevan a diferentes maneras de conducir una plantación, es por ello que el manejador debe de tomar la decisión en establecer la regulación de acuerdo a su entorno; la aplicación de diferentes técnicas de optimización propicia la determinación de optimo manejo silvicultura y operacional para la regulación con la definición de áreas y prescripción de manejo (Santos, 2008).

Los dos planos presentan la misma proyección de área de colecta forestal, regulándose entre sí a los 16 años (año futuro); en las edades, la proyección por RNA es más estandarizado que la proyección realizada por modelamiento de Clutter, donde se observa que la variable edad proyectada influye en la producción futura estimada, principal razón de la irregularidad en la proyección por Clutter. La variación en los valores de la tasa de interés y en el precio de la madera influencian en la solución de los modelos de regulación de la producción forestal (De Carvalho, 2015), tanto en los dos planos de proyección, la maximización del ingreso liquido es estable, sin embargo, no se consideraron otras restricciones como las sociales y ambientales, que usualmente generan variaciones en la maximización (Binoti, 2010).

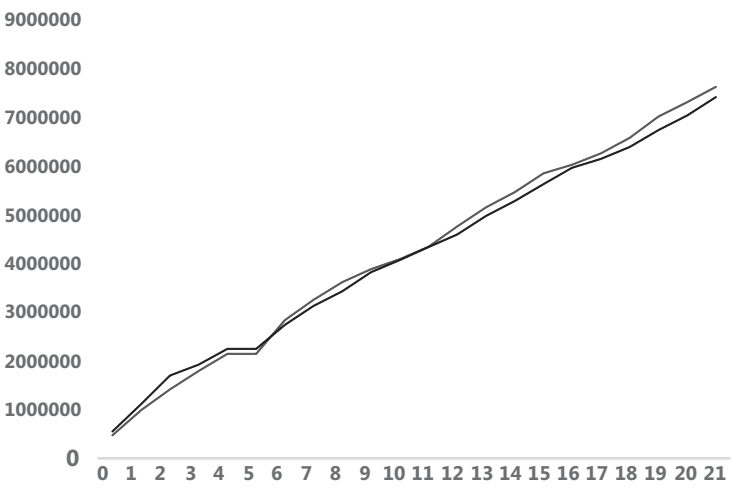

— FRECUENCIA DE RECEITA RNA — FRECUENCIA DE RECEITA CLUTTER

Figura 4. Ingreso acumulado prescrita por los modelos de regulación generados con los resultados de dos metodologías para proyección de producción, considerándose como la función objetivo en maximizar el ingreso líquido.

\section{CONCLUSIONES}

Los diferentes planos de proyección de producción tienen influencia en la regulación forestal, destacando las Redes Neuronales Artificiales como una alternativa más real y más confiable en la regulación forestal.

\section{AGRADECIMIENTOS}

Al Departamento de Ingeniería Forestal de la Universidade Federal de Viçosa y a la empresa Celulose Nipo-Brasileira S.A. por brindar las facilidades para realizar la presente investigación.

\section{REFERENCIAS BIBLIOGRÁFICAS}

Binoti, D. H. (2010). Estratégias de regulação de florestas equiâneas com vistas ao manejo da paisagem.

Binoti, M. (2012). Emprego de redes neurais artificiais em mensuração e manejo florestal (Tese Doutorado). Universidade Federal de Viçosa, Viçosa, MG, 2012

Binoti, M. L. (2010). Redes neurais artificiais para prognose da produção de povoamentos não desbastados de eucalipto (Dissertação Mestrado). Programa de Pós-Graduação em Ciência Florestal, Universidade Federal de Viçosa, Viçosa.

Bullinaria, J. A. (2014). Introduction to neural computation: notas de aula. Disponível 


\section{Regulación de la producción forestal}

em: $<$ http://www.cs.bham.ac.uk/ jxb/inc.ht $\mathrm{ml}>$.

Buongiorno, J., y Gilless, J. K. (2003). Decision methods for forest resource and management. California: Academic Press. $439 \mathrm{p}$.

Castro, R. R. (2007). Regulação de florestas eqüiâneas incluindo restrições de adjacência (Dissertação Magister Scientiae). Universidade Federal de Viçosa, Viçosa, MG.

Cavalcante, R. B. L. (2011). Planejamento de povoamentos de eucalipto com condicionantes hidrológicos: um estudo de caso em Eldorado do Sul-RS (Dissertação Mestrado). Universidade Federal do Rio Grande do Sul, Porto Alegre.

Clutter, J. L., Fortson, J. C., Pienaar, L. V., Brister, G. H., y Bailey, R. L. (1983). Timber management: a quantitative approach. John Wiley \& Sons, Inc.

Da Silva, Binoti, M. L., Binoti, D. H. B., Leite, H. G., Garcia, S. L. R., Ferreira, M. Z., Rode, R., y da Silva, A. A. (2014). Redes Neurais Artificiais Para Estimação Do Volume De Árvores. Revista Árvore, 38(2), 283-288.

Da Silva, G. F., Leite, H. G., Soares, C. P., y Da Silva, M. L. (2005). Influência de estimativas de produção de madeira em processos de regulação florestal utilizando programação linear. Ciência Florestal, 13(1), 57-72.

Davis, L. S.; Johnson, K. N. (1987). Forest management. New York: Mc Graw-Hill Book Company. 790p.

De Carvalho, K. H. A., Silva, M. L., Leite, H. G., \& Binoti, D. H. B. (2015). Influência da taxa de juros e do preço da madeira em modelos de regulação florestal. Pesquisa Florestal Brasileira, 35(82), 143-151.

Dykstra, D. P. (1984). Mathematical programming for natural resource management. New York: McGraw-Hill. 318 p.

Johnson, K. N., \& Scheurman, H. L. (1977). Techniques for prescribing optimal timber harvest and investment under different objectives--discussion and synthesis. Forest Science, 23(1), a0001-z0001.

Leuschner, W. A. (1984). Introduction to forest resource management. New York: John Willey \& Sons. 298p.
Magalhães, J. G. (2014). Prognose e Regulação de Florestas de eucalipto utilizando Modelo de Crescimento e Inteligência Computacional.

Monte, M. (2012). Inclusão de critérios sociais na modelagem matemática do planejamento florestal. 74 f. Tese (Doutorado em Ciência Florestal) - Universidade Federal de Viçosa, Viçosa, MG.

Rode, R., Leite, H. G., Oliveira, M. L., Binoti, D. H. B., Ribeiro, C. A., Souza, A. L. y Cosenza, D. N. (2015). Comparação da regulação florestal de projetos de fomento com áreas próprias de empresas florestais. Pesquisa Florestal Brasileira, 35(81), 11-19.

Rodrigues, F. L. (1997). Regulação de florestas equiâneas utilizando programação linear. 117 f. Dissertação (Mestrado em Ciência Florestal) - Universidade Federal de Viçosa, Viçosa, MG.

Santos, F. L. (2008). Regulação da produção de floresta de eucalipto submetida a desbaste e destinada a multiprodutos (Tesis de Mestrado). Universidade Federal de Viçosa, Viçosa, MG. 